\title{
Cytogenetic Biodosimetry using the Blood Lymphocytes of Astronauts
}

K. George, ${ }^{1}$ J. Rhone, ${ }^{2}$ L.J. Chappell ${ }^{3}$ and F. A. Cucinotta ${ }^{4}$

${ }^{1}$ Wyle, 1290 Hercules Drive, Houston, TX 77058, kerry.a.george@nasa.gov, ${ }^{2}$ University of Houston, 4800 Calhoun

Road, Houston, TX 77004, ${ }^{3}$ USRA, Division of Life Sciences, Houston TX 77058 and ${ }^{3}$ NASA, Lyndon B. Johnson Space Center, 2101 NASA Parkway, Houston TX 77058.

\begin{abstract}
Cytogenetic analysis of blood lymphocytes remains the most sensitive and reliable method available for in vivo assessment of the biological effects of exposure to radiation and provides the most informative measurement of radiation induced health risks. To date chromosome damage has been assessed in lymphocytes from more than 30 astronauts before and after they participated in long-duration space missions of three months or more on board the International Space Station. For all individuals, the frequency of chromosome damage measured within a month of return from space was higher than their prefight yield and biodosimetry estimates lie within the range expected from physical dosimetry. Biodosimetry data provides a direct measurement of space radiation damage, which takes into account individual radiosensitivity in the presence of confounding factors such as microgravity and other stress conditions. In contrast to physical measurements, which are external to body and require multiple devices to detect all radiation types all of which have poor sensitivity to neutrons, biodosimetry is internal and includes the effects of shielding provided by the body itself plus chromosome damage shows excellent sensitivity to protons, heavy ions, and neutrons. In addition, chromosome damage is reflective of cancer risk and biodosimetry values can therefore be used to validate and develop risk assessment models that can be used to characterize excess health risk incurred by crewmembers. A review of astronaut biodosimetry data will be presented along with recent findings on the persistence of space radiation induced chromosome damage and the cytogenetic effects of repeat long duration missions.
\end{abstract}

